P167 (continued)

Target Audience: Faculty, students, and community partners.

Theory, Prior Research, Rationale: Experiential learning has been well-documented to enhance student knowledge and professional preparation, but such highimpact activities can be challenging in high-enrollment courses.

Descripiton of Course and Curriculum: Undergraduate nutrition students at the University of Kentucky take a two-semester Life Cycle and Community Nutrition course sequence. In Part II of the course, students work in groups to develop a community programming intervention and evaluation plan. For spring 2017, a semester-long project and day-long field trip to rural Whitesburg, Kentucky, was included as part of the course to expose students to realworld challenges and opportunities in such a rural setting. Evaluation: Students completed anonymous surveys $(n=77)$ at the end of the semester. Survey results indicated that, on a Likert-scale of 1-7 with 1 being completely disagree and 7 being completely agree, students rated "The EL activity helped me to have a better understanding of my role as a community member" as a $5.3+/$ - 1.4, "I understand the attitudes of rural communities toward seeking and receiving healthcare" as $5.7+/-1.2$, and "I am sensitive to the psychological, cultural, and social factors that affect rural patients' health" as a $6.1+/$ - 0.9. On a similar scale with 7 being incredibly important, "How important do you think real-life applications are in your college coursework" was rated as a $6.5+/-0.9$.

Conclusions and Implications: Overall, students reported that the EL activity provided real-life application to the course and exposed them to opportunities working as a healthcare provider in a rural setting. Students provided valuable feedback on improvements that could be made to the project and field trip structure, which are being incorporated during the spring 2018 semester.

Funding: None.

\section{P168 Exploratory Thematic Analysis of Strategies to Improve the Healthfulness of the Food Pantry Environment}

Alexandra Bush-Kaufman, MPH, RDN,

alexandra.kaufman@wsu.edu, Washington State

University-Extension, 3602 Pacific Avenue, Suite 200,

Tacoma, WA 98418; Karen Barale, MS, RDN, FAND;

Marie Walsh, MPH, Colorado State University-Extension; Rebecca Sero, PhD, Washington State University-

Extension

Background (Background, Rationale, Prior Research, and/or Theory): Private food assistance can provide up to $25 \%$ of low-income Americans' monthly diets. Improving the healthfulness of food pantry environments may improve the health of people living in poverty and potentially reduce the prevalence of dietrelated chronic diseases. Anecdotal evidence of existing strategies provides little insight on the interventions that influence pantry environments.
Objective: Identify the processes food pantries and food banks implement to promote healthy environmental strategies. Characterize the challenges pantries and food banks face during these processes.

Study Design, Setting, Participants, Intervention: Qualitative semi-structured phone interviews were conducted with key informants representing food pantry managers, food bank personnel, and community partners $(n=42)$ selected through purposive and snowball sampling of the western United States.

Outcome Measures and Analysis: Interviews were transcribed and analyzed using Atlas.ti 8. Data were coded using a hierarchical coding scheme. Descriptive codes included "strategy codes" defining the program and "process codes" that outlined the characteristics of initiation, challenge, and evaluation. Cross-coding of process codes was applied to strategies. Exploratory thematic analysis and inductive modeling was completed to build models of resulting interventions.

Results: Four dimensions of a healthy pantry environment were discovered: food, emotional, physical, and informational. The emotional and physical dimensions were closely related and generated eight "healthy environment strategies." Approaches addressing the informational dimension generated four "service strategies." Findings resulted in eight initiation characteristics (e.g. external support), 11 challenges (e.g. high client volume), and seven evaluation methods (e.g. survey).

Conclusions and Implications: The strategy models identified can benefit future program planning and evaluation for interventions within the food pantry environment. Successful program evaluation may ensure that effective strategies are carried out to improve diet quality and health outcomes for pantry clients.

Funding: NIFA Regional Nutrition Education, Obesity Prevention Center of Excellences Grant.

\section{P169 Exploring Quality Assessment in Community-Based Nutrition Education}

Ghaffar Hurtado Choque,PhD, ahurtado@umd.edu, University of Maryland, 4200 Valley Drive, 1142E School of Public Health, College Park, MD 20742;

Silvia Alvarez de Davila, PhD, University of Minnesota; Nancy Brewster, PhD

Background (Background, Rationale, Prior Research, and/or Theory): Quality of program delivery is a major component in community-based nutrition education and is also considered "an art and a science"; and while this is associated with positive outcomes, little is known about how the quality of implementation is assessed in community nutrition programs. Padres Preparados, Jóvenes Saludables (PPJS) is a federally funded, longitudinal prevention intervention. We focus on strengthening family-skills of immigrant Latino parents (especially fathers) of adolescents, aimed to promote healthy energy balancerelated behaviors. 
P169 (continued)

Objective: We explored quality of implementation during a pilot evaluation in spring 2017, using a multiprong approach: principles-based fidelity, attendance, and participant reaction: interest, relevance, comfort level, and satisfaction.

Description: We conducted live observation of a subset of sessions with a check-list of "core concepts". Facilitators answered questions regarding participant engagement and understanding of concepts presented. Participants completed a session evaluation that assessed understanding of core concepts presented (knowledge), and facilitator rapport. We aimed to assess adherence but also other site-specific nuances associated with learning and key outcomes.

Evaluation: Of the 13 participating families (fathers), most attended six sessions. In total, parents completed 109 after-session (one-page) evaluation forms. Most (90\%) indicated class sessions were somewhat or very useful, somewhat or very interesting, and that participants felt comfortable sharing opinions. Most (94\%) of their responses rated facilitators as supportive and as having addressed participants' needs and interests.

Conclusions and Implications: This process evaluation findings illustrate quality implementation assessment. Overall facilitators had high levels of facilitation skills. The principle-based fidelity addressed the necessity, not only to capture adherence, but also whether the program has been able to achieve the desired results. It also provided insight to implementation that allows for adaptability to "real life" situations without loss of effectiveness. However, it called for a highly-skilled evaluator and developing the indicators of that can be broadly used in community settings.

Funding: USDA.

\section{P170 Exploring the Use of Online Learning in Postsecondary Nutrition Education Courses: A Systematic Review}

Amy Spielmaker, BS, RDN, amykspielmaker@gmail.com, Western Oregon University, 345 Monmouth Avenue N, Monmouth, OR 97361; Megan Patton-López, PhD, RDN; Zubaida Qamar, PhD, University of California, San Francisco; Mallory Koenings, PhD, RDN, USDA, National Institute of Food and Agriculture; Brandy-Joe Milliron, PhD, Drexel University; Marissa Burgermaster, PhD, Columbia University

Background (Background, Rationale, Prior Research, and/or Theory): The internet and new information technologies have transformed education. Nutrition education is among many postsecondary subjects being taught using online instructional methods. With increasing demand for flexible coursework to prepare nutrition professionals, educators will benefit from a synthesis of what is known about effective online learning for nutrition education courses.

Objective: To investigate the current use of online instruction in undergraduate and graduate nutrition courses, determine course effectiveness, and identify practical recommendations for educators.

Study Design, Setting, Participants, Intervention: We systematically reviewed studies via a comprehensive search of major databases using a standardized search strategy. Studies selected for inclusion were those that evaluated the knowledge and/or perceptions of undergraduate or graduate students in postsecondary nutrition education courses delivered completely or primarily online. We considered both experimental and epidemiological study designs.

Outcome Measures and Analysis: We extracted data on course instructional design, study design, participants, and outcomes from the studies meeting our inclusion criteria. Four reviewers independently assessed study quality using the Nutrition Evidence Library Bias Elimination Tool. Results: Nineteen of 5,803 search results met the inclusion criteria. Most involved asynchronous online courses in health professional studies (e.g. dietetics, nursing, medicine). Few studies employed an experimental design and many lacked details regarding the course's instructional design. Among studies with a comparison group $(n=7)$, there were no differences in nutrition knowledge between online and face-to-face learners. Results were inconclusive regarding students' perceptions.

Conclusions and Implications: Current reports suggest that neither online nor face-to-face nutrition courses are superior and students' experiences with online learning vary. Recommendations for educators include being engaged with the online class during discussions, providing regular feedback, becoming comfortable with different technologies, and developing course content that facilitates discovery learning. The heterogeneous methods and limited descriptions of instructional design we identified highlight the need for research that elucidates effective approaches to online learning in postsecondary nutrition education courses.

Funding: None.

\section{P171 Field Testing of Complementary Feeding and Nutrition Education Intervention in Selected Philippine Villages}

Julieta B. Dorado, juliedorado2015@gmail.com, Department of Science and Technology_Food and

Nutrition Research Institute, General Santos Avenue, Taguig City, Metro Manila, 01633, Philippines; Clarita R. Magsadia, MS; Rowena V. Viajar, MS; Chona F. Patalen, MS; Glenda P. Azana;

Mario V. Capanzana, PhD

Background (Background, Rationale, Prior Research, and/or Theory): In response to the problem of malnutrition in the Philippine countryside, the Department of Science and Technology, through the Food and Nutrition Research Institute (DOST-FNRI), developed a package of interventions which combines complementary feeding of young children and nutrition education of mothers and caregivers. 